\title{
Co-regulation and multilocus determinants of gene expression in
} humans

\author{
Berit Kerner*1, Julia N Bailey² and Rita M Cantor ${ }^{3}$
}

Address: ${ }^{1}$ Center for Neurobehavioral Genetics, Department of Psychiatry and Biobehavioral Sciences, Semel Institute for Neuroscience and Human Behavior, 695 Charles E. Young Drive South, Los Angeles, California 90095-1761, USA, ² Center for Primate Neuroethology, Department of Psychiatry and Biobehavioral Sciences, Semel Institute for Neuroscience and Human Behavior, 695 Charles E. Young Drive South, Los Angeles, California 90095-1761, USA and 32Departments of Human Genetics and Pediatrics, David Geffen School of Medicine at UCLA, 695 Charles E. Young Drive South, Los Angeles, California 90095-7088, USA

Email: Berit Kerner* - bkerner@mednet.ucla.edu; Julia N Bailey - JBailey@mednet.ucla.edu; Rita M Cantor - rcantor@mednet.ucla.edu

* Corresponding author

from Genetic Analysis Workshop 15

St. Pete Beach, Florida, USA. II-15 November 2006

Published: 18 December 2007

BMC Proceedings 2007, I(Suppl I):S88

This article is available from: http://www.biomedcentral.com/I753-656I/I/SI/S88

(c) 2007 Kerner et al; licensee BioMed Central Ltd.

This is an open access article distributed under the terms of the Creative Commons Attribution License (http://creativecommons.org/licenses/by/2.0), which permits unrestricted use, distribution, and reproduction in any medium, provided the original work is properly cited.

\begin{abstract}
Background: The regulation of gene expression is an emerging area of investigation. Increased knowledge can deepen our understanding of the genetic contributions to variations in complex traits. The purpose of this study is to explore the feasibility of detecting regulatory elements of gene expression with multivariate analyses.
\end{abstract}

Methods: Peripheral blood lymphocyte expression levels of 30 genes on chromosome 5 and a single gene, $D E A D$, on chromosome 22 were analyzed in single-point variance-component linkage analyses in multiplex families to identify putative regulatory regions. To explore the possibility of regulatory regions having individual relationships with the expression levels of a single gene, we utilized stepwise regression. To explore the possibility of pleiotropy of a single regulatory locus for multiple genes, bivariate linkage analysis was applied.

Results: Twenty-one loci were linked to five expression levels. The two most significant were for the known region on chromosome 22 (LOD = 4.62). On chromosome 5 a LOD of 4.57 was found for the gene leukocyte-derived arginine aminopeptidase (LRAP) with a single-nucleotide polymorphism (SNP) within $5 \mathrm{Mb}$. Both genes showed evidence of linkage to multiple SNPs. When 194 family members were treated as independent, stepwise regression identified fewer single-nucleotide polymorphisms with significant predictive values $(p<0.05)$, providing evidence for multiple regulatory regions of unequal effect. However, when corrections for non-independence were applied these results could no longer be detected.

Conclusion: The complex nature of gene regulation can be explored by linkage analysis with single-nucleotide polymorphisms followed by multivariate methods to explore co-regulation. 


\section{Background}

Regulation of gene expression in human peripheral blood lymphocytes is not well understood. Most knowledge comes from the study of individual genes and their nearby regulatory elements. Taking this approach, a relatively small number of differentially expressed genes has been identified, and their regulatory elements found to have a major effect on gene expression $(>50 \%)$.

However, emerging evidence indicates that gene expression is regulated by a network of elements that are both close to the gene and at large distances. Studies in flies, yeast, and mice suggest that most genes are regulated by many regulatory elements with small effects and these complicated interactions may be difficult to detect with current methods of analysis [1]. High-throughput methods for single-nucleotide polymorphism (SNP) genotyping and detection of expression levels allow us to further investigate these mechanisms.

We report a study designed to explore the feasibility of detecting regulatory elements of gene expression with multivariate analyses.

\section{Methods}

The Genetic Analysis Workshop I5 (GAWI5) sample

Gene expression data from lymphoblastoid cell lines of 194 individuals from 14 three-generation Centre d'Etude du Polymorphisme Humain families obtained using an Affimetrix Human Focus Array were provided. This study was approved by the Institutional Review Board at UCLA.

\section{Selection of genes and SNPs}

To apply our method we focused our analysis on 90 gene expression traits for those genes residing on chromosome 5q. One trait on chromosome 22 with a known significant linkage signal to a SNP in close proximity to the gene was selected as well [2]. For these genes, we tested the normality of expression levels, and estimated their heritability using the SOLAR software [3]. We also included sex as a covariate in the genetic model to test its significance for the expression levels of the genes. Only traits with a heritability $\geq 0.2$ and skewness and kurtosis estimates within the normal range were retained. Thirty traits on chromosome $5 \mathrm{q}$ and chromosome 22 met these criteria. Four SNPs on chromosome 5 had a Mendelian error in two families and eight SNPs on chromosome 22 had Mendelian errors in four families. Those families with genotypes with Mendelian inconsistencies were eliminated for the particular SNP.

\section{Linkage analysis}

Single and bivariate trait single-point variance-component (VC) quantitative trait linkage (QTL) analyses were performed using the SOLAR software on gene expression levels for the 31 genes selected. Given the relatively small number of pedigrees and the exploratory nature of these studies, a LOD of 2.0 was used as the threshold for linkage.

\section{Multivariate analyses}

In cases in which multiple SNPs were linked to an expression trait, stepwise multivariate regression analysis was used to identify loci that were the strongest predictors of the expression levels, and to understand if all loci contributed equally [4]. In stepwise regression the SNP genotypes for each individual were used as explanatory variables after recoding them in a co-dominant fashion. Sex was included in the analysis to determine its possible significance. Analyses were conducted using SAS software version 9.1 [5]. A significance level of 0.05 was set as a criterion for entry into the model, as well as for remaining in the model. Because our data were collected in families that shared genetic information, they were not uncorrelated. We therefore took non-independence of the observations into account by using the final model from the stepwise regression and calculating a robust standard error using Proc Surveyreg. This procedure computes the regression coefficient estimation by generalized leastsquares estimation using element-wise regression and the Taylor expansion theory for estimating sampling errors of estimation based on complex sample designs [5].

\section{Test for pleiotropy}

To test for the possibility that a single gene (allele) influences the expression levels of multiple traits, we extended the univariate genetic analyses to a bivariate analysis in which the bivariate phenotypes are modeled as the outcome and genetic and environmental correlations can be estimated as described in detail by Almasy et al. [6]. A model in which all parameters are estimated is compared with a model in which the genetic correlation is constrained to zero to test for pleiotropic effects. The significance of this test implies a common set of genes contributing to the variance in the two traits. This model can be extended to incorporate SNP data. We can then test for linkage by comparing likelihoods of the model with and without the genetic data. Twice the difference of the $\log$ likelihoods of the two models is distributed asymptotically as a chi-square with 1 degree of freedom. A bivariate analysis was conducted in SOLAR for those genes where expression levels showed evidence of co-regulation by a single common SNP in order to test for the possibility that such a joint analysis would increase the power to detect linkage.

\section{Results \\ Linkage and multivariate analysis}

Out of the 30 heritable expression level traits on chromosome 5,4 showed evidence of linkage at a LOD score $>2$ 
to 11 SNPs on the same chromosome (Table 1 ). The heritability estimates of these traits ranged from 0.41 to 0.61 and sex as a covariate did not have a significant effect. The strongest linkage signal (LOD score of 4.6) was obtained between variation in expression levels of the gene LRAP and the SNP rs18224780, located approximately 5 megabase pairs $(\mathrm{Mb})$ away from the gene locus.

Expression levels of two genes on chromosome 5 showed evidence of linkage to multiple SNPs (Table 1), and in these cases, stepwise regression was used to select the most significant predictors of expression. For the gene $L R A P$, the SNP rs 1824780 was significant at the 0.05 level, however, when we took the non-independence of the observations into account by using the final model from the stepwise regression and calculating robust standard errors using Proc Surveyreg, those SNPs were no longer significant (Table 2). The second gene, G protein-coupled receptor kinase $6(G R K \sigma)$, did not reveal significant results in stepwise regression.

The gene DEAD (DDX17), located on chromosome 22, gave multiple strong linkage signals along the entire long arm of chromosome 22 (Figure 1, Table 1). The highest LOD score (LOD = 4.62 at SNP rs132404) was located approximately $9 \mathrm{Mb}$ away from the location of the gene.
We included all SNPs with evidence for linkage (LOD > 2) in a stepwise regression analysis (Table 2). Only two of these SNPs (rs760482 and rs80533) remained in the model at $p<0.05$. These SNPs were located within $5 \mathrm{Mb}$ of the gene locus. None of these three genes had significant sex effects in the stepwise regression analysis.

\section{Pleiotropy}

The results of the combined polygenic VC linkage analysis showed that the genes Treacher Collins-Franceschetti syndrome 1 (TCOF1) and GRK6 share a strong and significant genetic correlation $\left(\rho_{g}=0.94 \pm 0.07, p<0.0001\right)$, implying these two expression traits may share susceptibility genes at that locus, and that these two expression traits may be regulated by the same SNP. The environmental correlation for these expression levels $\left(\rho_{e}=0.58 \pm 0.09\right.$, $p=0.00002$ ) was also significant. Single-point linkage analysis, which includes a major gene for both traits jointly, showed slight improvement in the LOD score at the linked SNP for trait TCOF1 (LOD $=2.3$ for the trait alone compared to LOD $=2.5$ in the joint analysis) and a slight decrease in the LOD score for trait GRK6 (LOD $=2.8$ compared to $\mathrm{LOD}=2.5$ in the joint analysis) (Figure 2). Thus, we have no evidence that a single SNP contributes to the expression levels of these two genes.

Table I: Expression traits with linkage to SNPs on Chromosomes 5 and 22

\begin{tabular}{|c|c|c|c|c|c|c|c|}
\hline $\begin{array}{c}\text { Expression } \\
\text { Trait }\end{array}$ & Trait name & Chr & Location (Mb) & Heritability & LOD score & SNP & Location (Mb) \\
\hline \multirow[t]{6}{*}{ LRAP } & Leukocyte-derived arginine aminopeptidase & $5 q 15$ & 96.2 & 0.59 & 4.6 & rs I 824780a & 99.7 \\
\hline & & & & & 3.4 & rs|973172 & 99.7 \\
\hline & & & & & 3.1 & rs 1824777 & 99.6 \\
\hline & & & & & 2.8 & rs421508 & 83.3 \\
\hline & & & & & 2.8 & rs 1464765 & 111.6 \\
\hline & & & & & 2.3 & rs 1020720 & 80.5 \\
\hline$S A R I B$ & SARI gene homolog B (S. cerevisiae) & $5 q 31.1$ & 133.9 & 0.45 & 2.1 & rs428538 & 104 \\
\hline TCOFI & Treacher Collins-Franceschetti syndrome I & $5 q 32-33$ & 149.7 & 0.41 & 2.3 & rs984091 & 66.5 \\
\hline \multirow[t]{3}{*}{ GRK6 } & G protein-coupled receptor kinase 6 & $5 q 35$ & 176.7 & 0.61 & 2.8 & rs984091 & 66.5 \\
\hline & & & & & 2.5 & rs334908 & 34.7 \\
\hline & & & & & 2.2 & rs506056 & 39.8 \\
\hline \multirow[t]{10}{*}{$D D X I 7$} & DEAD (Asp-GI-Ala-Asp) box polypeptide 17 & $22 q 13$ & 37.2 & 0.59 & 4.6 & rs 132404 & 43.4 \\
\hline & & & & & 4.1 & rs80533 & 39.4 \\
\hline & & & & & 3.7 & rs 141418 & 32.8 \\
\hline & & & & & 3.5 & rs738733 & 46.6 \\
\hline & & & & & 3.3 & rs760482 & 37.5 \\
\hline & & & & & 3 & rs734139 & 35.9 \\
\hline & & & & & 2.8 & rs72859l & 46.5 \\
\hline & & & & & 2.3 & rs916336 & 34.9 \\
\hline & & & & & 2.2 & rs2032474 & 32 \\
\hline & & & & & 2 & rs713912 & 42.3 \\
\hline
\end{tabular}

aBold text indicates SNPs that remained in the stepwise regression analyses. 
Table 2: Results of stepwise regression

\begin{tabular}{llcc}
\hline Gene & SNP & F-Value & $\operatorname{Pr}>\mathrm{F}$ \\
\hline LARP & rs1824780 & 4.93 & 0.03 \\
DDXI7 & rs760482 & 9.23 & 0.003 \\
& rs80533 & 4.02 & 0.046 \\
\hline
\end{tabular}

\section{Discussion}

In an exploratory linkage study of 31 expression traits on chromosomes 5 and 22, we found significant linkage signals for two traits, LRAP and DDX17, with SNPs in close proximity to the gene loci (cis). Sex as a covariate was not significant in our analysis. Other GAW15 groups who analyzed these data reported similar results $[7,8]$. Those two expression traits also showed evidence of linkage to multiple SNPs at considerable distances away from the gene locations (trans). In an evaluation of co-regulation, the SNPs did not remain significant in a stepwise regression when the non-independence of the data due to the family structure was taken into account. Evidence for potential pleiotropy was not supported by bivariate QTL analysis. Unfortunately, this study is limited by the number of families we could include in the analyses.

\section{Conclusion}

We demonstrate here that the complex nature of gene regulation can be explored by linkage analysis with SNPs followed by multivariate methods to explore co-regulation.

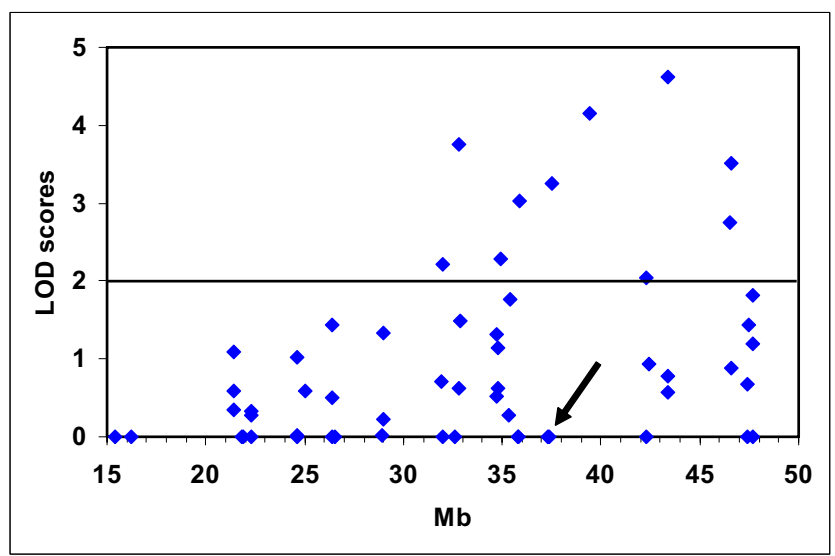

\section{Figure I}

Single-point QTL analysis for the trait gene expression level of the gene DEAD (DDXI7). The horizontal axis shows the distance along chromosome 22 in megabase pairs (Mb). The vertical axis shows the LOD scores. The gene itself, located on chromosome $22 \mathrm{ql} 3$ at $37.2 \mathrm{Mb}$, is indicated by the arrow.

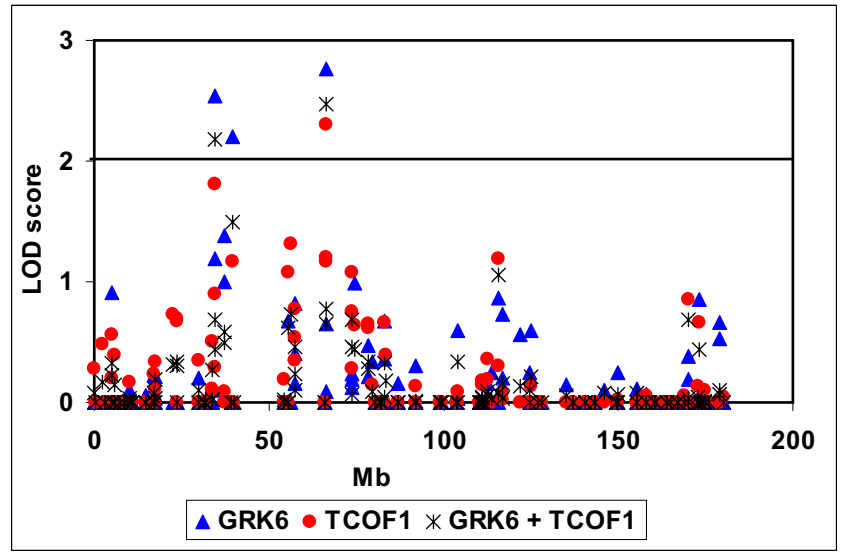

Figure 2

Univariate and bivariate single-point QTL analyses for the traits gene expression levels of the genes GRK6 and TCOFI along chromosome 5. Gene expression levels of the genes TCOFI and GRK6 are linked to the same SNPs. *, joint single-point QTL analysis. The distance along chromosome 5 in megabase pairs $(\mathrm{Mb})$ is on the horizontal axis. LOD scores of QTL analyses are on the vertical axis.

\section{Competing interests}

The author(s) declare that they have no competing interests.

\section{Acknowledgements}

This work was supported in part by the Statistical Core (RMC) of NIH Program Project HL-2848, and NIMH grant K08 MH074057-0I to BK.

This article has been published as part of BMC Proceedings Volume I Supplement I, 2007: Genetic Analysis Workshop I5: Gene Expression Analysis and Approaches to Detecting Multiple Functional Loci. The full contents of the supplement are available online at http://www.biomedcentral.com/ $\underline{|753-656| / \mid \text { ? issue=SI. }}$.

\section{References}

I. Stamatoyannopoulos JA: The genomics of gene expression. Genomics 2004, 84:449-457.

2. Morley M, Molony CM, Weber T, Devlin JL, Ewens KG, Spielman RS, Cheung VG: Genetic analysis of genome-wide variation in human gene expression. Nature 2004, 430:743-747.

3. Almasy L, Blangero J: Multipoint quantitative trait linkage analysis in general pedigrees. Am J Hum Genet 1998, 62:1 I98-1211.

4. Hocking RR: The analysis and selection of variables in linear regression. Biometrics 1976, 32: I-50.

5. SAS Institute Inc: SAS Online Documentation, Version 9.I Cary, NC: SAS Institute Inc; 2004.

6. Almasy L, Dyer TD, Blangero J: Bivariate quantitative trait linkage analysis: pleiotropy versus co-incident linkages. Genet Epidemiol 1997, I 4:953-958.

7. Sung YJ, Di Y, Fu AQ, Rothstein JH, Sieh W, Tong L, Thompson EA, Wijsman EM: Comparison of multipoint linkage analyses for quantitative traits in the CEPH data: parametric LOD scores, variance components LOD scores, and Bayes factors. BMC Proc 2007, I (Suppl I):S93. 
8. Rangrej J, Beyene J, Hu P, Paterson AD: Sex, age, and generation effects on genome-wide linkage analysis of gene expression in transformed lymphoblasts. BMC Proc 2007, I (SuppI I):S92.

Publish with Bio Med Central and every scientist can read your work free of charge

"BioMed Central will be the most significant development for disseminating the results of biomedical research in our lifetime. " Sir Paul Nurse, Cancer Research UK

Your research papers will be:

- available free of charge to the entire biomedical community

- peer reviewed and published immediately upon acceptance

- cited in PubMed and archived on PubMed Central

- yours - you keep the copyright

Submit your manuscript here:

http://www.biomedcentral.com/info/publishing_adv.asp 